\section{POS1141 ASSESSING RELEVANT JOINTS FOR MONITORING CPPD DISEASE: A SYSTEMATIC LITERATURE REVIEW OF IMAGING TECHNIQUES BY THE OMERACT ULTRASOUND - CPPD SUBGROUP}

A. Adinolfi ${ }^{1}$, S. Sirotti ${ }^{2}$, G. Sakellariou ${ }^{3}$, E. Cipolletta ${ }^{4}$, E. Filippucci ${ }^{4}$, F. Porta ${ }^{5}$, P. Sarzi-Puttini ${ }^{2}$, C. A. Scirè ${ }^{6}$, H. Keen ${ }^{7}$, P. Mandl ${ }^{8}$, G. Mouterde ${ }^{9}$, C. Pineda ${ }^{10}$, L. Terslev ${ }^{11}$, M. A. D'agostino ${ }^{12}$, A. lagnocco ${ }^{13}$, G. Filippou ${ }^{2}$ on behalf of Omeract Ultrasound working group, subgroup CPPD. ${ }^{1}$ ASST Grande Ospedale Metropolitano Niguarda, Rheumatology Unit, Milan, Italy; ${ }^{2}$ Luigi Sacco University Hospital, Rheumatology Department, Milan, Italy; ${ }^{3}$ stituti Clinici Scientifici Maugeri IRCCS, University of Pavia, Pavia, Italy; ${ }^{4}$ Università Politecnica delle Marche, Rheumatology Unit, Department of Clinical and Molecular Sciences, Ancona, Italy; ${ }^{5}$ Institute of Neurosciences, Neurosciences, Florence, Italy; ${ }^{6}$ SIR Epidemiology, Research Unit, Milan, Italy; ${ }^{7}$ The University of Western Australia, Medicine and Pharmacology Department, Murdoch, Perth, Australia; ${ }^{8}$ Medical University Vienna, Internal Medicine 3, Division of Rheumatology, Vienna, Austria; ${ }^{9}$ University of Montpellier, Rheumatology, Montpellier, France; ${ }^{10}$ Instituto Nacional de Rehabilitacion, Rheumatology Department, Mexico City, Mexico; ${ }^{11}$ Copenhagen University, Center for Rheumatology and Spine Diseases, Rigshospitalet, Copenhagen, Denmark; ${ }^{12}$ Università Cattolica del Sacro Cuore, Policlinico Universitario Agostino Gemelli IRCSS, Rheumatology Department, Rome, Italy; ${ }^{13}$ Università degli Studi di Torino, Rheumatology Unit, Department of Clinical and Biological Sciences, Torino, Italy

Background: Imaging has been extensively used for the Calcium Pyrophosphate Deposition Disease (CPPD) diagnosis but the prevalence of joint calcifications at imaging in CPPD patients has not been thoroughly assessed.

Objectives: This systematic literature review (SLR) is aimed to estimate the prevalence of calcium crystal deposition in peripheral joints by imaging in suspected or definite CPPD patients to establish relevant joints for CPPD monitoring Methods: After defining PICOs, Pubmed and Embase were searched from inception to October 2020 for identifying studies that evaluated the use of Conventional Radiography (CR), Ultrasound (US), Computed Tomography (CT) and Dual Energy Computed Tomography (DECT) in detecting calcifications at peripheral joints in patients with defined or probable CPPD. Search strategies based on MeSH terms and free text were applied. Six reviewers independently screened titles and abstracts, eligible article full texts were evaluated for inclusion and data extraction.

Results: The SLR identified 1149 manuscripts. 524 papers entered in the full text evaluation and 181 articles were finally included. Considering excluded abstracts and full texts, 23 and 17 were excluded as duplicate, 156 and 9 for language, 171 and 48 for population, 171 and 20 for intervention, 57 and 117 for outcome, 47 and 70 for study type and 76 for full text unavailability. Among included, 41 papers considered patients with definite CPPD with a total population of 1239 patients, 908 cases and 331 controls and 140 papers referred to patients with suspected CPPD with a total population of 26785 patients, 2896 affected by CPPD and 23889 controls. The results about all joints are summarized in the Table 1. In patients with definite diagnosis, knee and wrist are the joints with the highest prevalence of calcifications at all imaging types, followed by hip and acromion-clavicular joint while in patients with suspected CPPD, the knee is the most prevalent followed by the wrist, hip and ankle (only sites with more than 50 patients assessed considered). The hand joints are characterized by CPPD lowest prevalence at imaging. Bilateral imaging findings varies depending on the technique used, the site and the patient's type.
Conclusion: According to the results of this SLR, knees and wrists could be the sentinel joints for CPPD detection by imaging.

Disclosure of Interests: None declared.

DOI: 10.1136/annrheumdis-2021-eular.3153

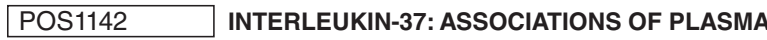 LEVELS AND GENETIC VARIANTS IN GOUT}

A. Navrátilová ${ }^{1,2}$, V. Voclonová ${ }^{1}$, H. Hulejova ${ }^{1}$, L. Andres Cerezo ${ }^{1,2}$, J. Zavada $^{1,2}$, K. Pavelka ${ }^{1,2}$, L. Šenolt ${ }^{1,2}$, B. Stiburkova ${ }^{1,3,4} \cdot{ }^{1}$ Institute of rheumatology, Department of Experimental Rheumatology, Prague, Czech Republic; ${ }^{2}$ First Faculty of Medicine Charles University, Department of Rheumatology, Prague, Czech Republic; ${ }^{3}$ First Faculty of Medicine Charles University, Department of Pediatrics and Adolescent Medicine, Prague, Czech Republic; ${ }^{4}$ General University Hospital in Prague, Department of Pediatrics and Adolescent Medicine, Prague, Czech Republic

Background: IL-37, recently characterized IL-1 family member, has anti-inflammatory effects by suppression of IL-1 1 and other proinflammatory cytokines. In this study we investigated the effects of genetics variants in IL-37 link with IL-37 plasma levels in a cohorts of patients with hyperuricemia/gout.

Objectives: The aim of this study was to determine the association of IL-37 gene polymorphism and plasma IL-37 levels in patients with hyperuricemia and gout. Methods: The cohorts consisted of 50 control subjects, 50 subjects of primary hyperuricemia, 50 subjects of primary gout, 28 subjects of tophaceous gout and 19 subjects of acute gout flare. The analyzed cohorts were selected from a previously reported set of 250 hyperuricemia/gout patients and 132 normouricemic subjects (1) according to the descending level of serum urate. All coding regions and intron-exon boundaries of IL-37, exon 1-5, were amplified and sequenced directly. Comparisons of presence/absence of identified variants was performed using P-values binomial test. Levels of plasma IL-37 were measured using Enzyme-Linked ImmunoSorbent Assay. All tests were performed in accordance with standards set by the institutional ethics committees, which approved the project in Prague (no.6181/2015).

Results: We identified 12 IL-37 genetic variants: five intron (rs28947188, rs2466448, rs3811045, rs3811048, rs2708944), and seven non-synonymous allelic variants (rs3811046, rs3811047, rs2708943, rs2723183, rs2723187, rs2708947, rs27231927). Minor allele frequency (MAF) of those variants in European population from ExAC databases were used for comparison. Our data showed that the rs28947188, rs3811045, rs3811046, rs3811047, rs2723187, rs2708947, and rs27231927 variants were under-represented in the Czech hyperuricemia, gout, and tophaceous gout cohort compared with the control cohort and general European population $(P=0.0082-0.0395)$.

The levels of plasma IL-37 were significantly higher in patients with tophaceous gout compared to control subjects ( $P$ 0.0329) whereas no changes were observed in subjects with primary hyperuricemia, primary gout or acute gout flare compared to control subjects. However, IL-37 was elevated in cohorts of patients with gout, tophaceous gout and acute gout flare compared to primary hyperuricemia subjects ( $\mathrm{P} 0.0198,0.0005,0.0099$; respectively).

Conclusion: Although further analyzes are needed to elucidate the role of IL-37 in the gout, our results show that genetic variants in anti-inflammatory cytokine IL-37 are probably implicated in the pathogenesis of gout.

\section{REFERENCES:}

[1] Toyoda Y, et al. Functional characterization of clinically-relevant rare variants in ABCG2 identified in a gout and hyperuricemia cohort. Cells. 2019 Apr $18 ; 8(4)$.

Table 1. Definite CPPD Diagnosis

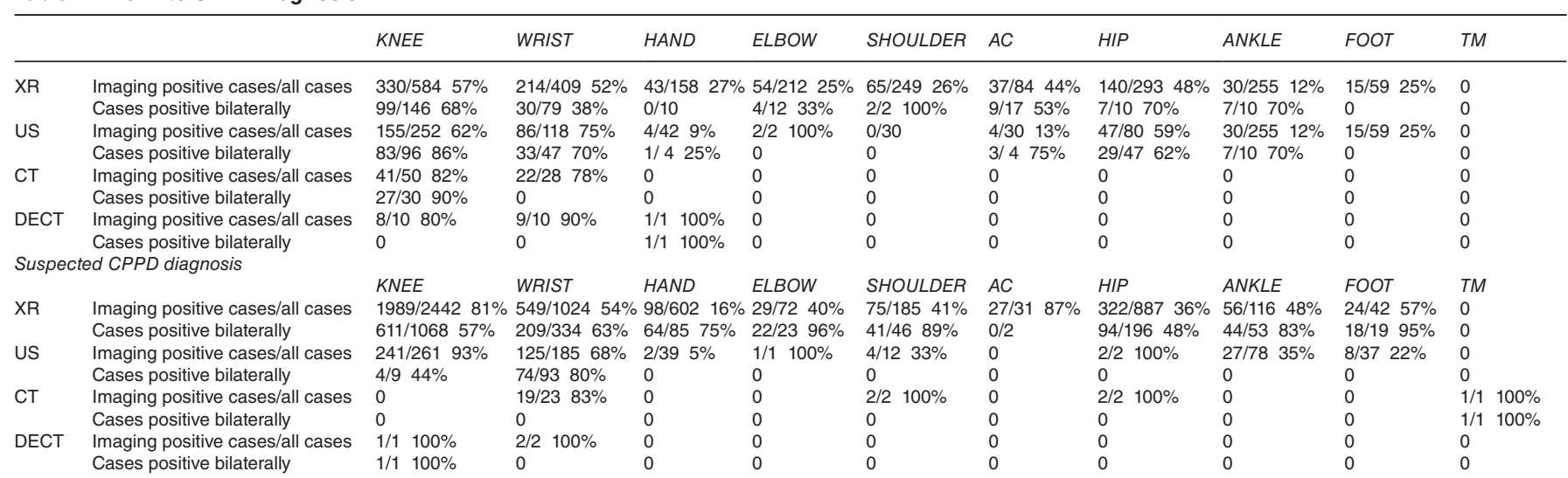

Table 1 For each joint, are summarized the ratio between positive joints and overall evaluated joints and the ratio between the joints positive bilaterally and overall joints evaluated bilaterally.AC Acromion Clavicular, TM temporo mandibular 